\title{
ON LEFT ABSOLUTELY FLAT BANDS
}

\author{
SYDNEY BULMAN-FLEMING AND KENNETH MCDOWELL
}

(Communicated by Bhama Srinivasan)

\begin{abstract}
A semigroup $S$ is called (left, right) absolutely flat if all of its (left, right) $S$-sets are flat. Let $S=\bigcup\left\{S_{\gamma}: \gamma \in \Gamma\right\}$ be the least semilattice decomposition of a band $S$. It is known that if $S$ is left absolutely flat then $S$ is right regular (that is, each $S_{\gamma}$ is right zero). In this paper it is shown that, in addition, whenever $\alpha, \beta \in \Gamma, \alpha<\beta$, and $F$ is a finite subset of $S_{\beta} \times S_{\beta}$, there exists $w \in S_{\alpha}$ such that $(w u, w v) \in \theta_{R}(F)$ for all $(u, v) \in F\left(\theta_{R}(F)\right.$ denotes the smallest right congruence on $S$ containing $F$ ). This condition in fact affords a characterization of left absolute flatness in certain classes of right regular bands (e.g. if $\Gamma$ is a chain, if all chains contained in $\Gamma$ have at most two elements, or if $S$ is right normal).
\end{abstract}

1. Introduction. Let $S$ be a band. Then $S$ is a semilattice of rectangular bands, and if $S$ is left absolutely flat then each rectangular component must be a right zero band ([3], or an easy adaptation of [1, Theorem 4.3]). Therefore every left absolutely flat band is right regular (the variety of right regular bands is defined by the equation $x y x=y x$ ). In the case where $S$ is right normal, $S$ is left absolutely flat if and only if the nonidentity structure maps are constant [2]. If $S$ is an arbitrary band a characterization of left absolute flatness which makes no reference to $S$-sets is not known, and the problem of finding one appears to be difficult. The main goal of this paper is to establish a condition which is necessary for a band to be left absolutely flat. This condition is also sufficient in special cases.

2. A necessary condition for left absolute flatness. For any semigroup $S$, $S$-Ens (respectively, Ens-S) will denote the class of all left (right) $S$-sets and $S^{1}$ will represent the monoid obtained by adjoining a new identity element 1 to $S$. Let $A \in$ Ens- $S, a, a^{\prime} \in A, B \in S$-Ens, and $b, b^{\prime} \in B$. Following Lemma 1.2 of $[\mathbf{1}]$ it is easy to show that $a \otimes b=a^{\prime} \otimes b^{\prime}$ in $A \otimes B$ if and only if there exist $a_{1}, \ldots, a_{n} \in A$, $b_{2}, \ldots, b_{n} \in B, s_{1}, \ldots, s_{n}, t_{1}, \ldots, t_{n} \in S^{1}$ such that

$$
\begin{array}{rlrl}
a & =a_{1} s_{1} & \\
a_{1} t_{1} & =a_{2} s_{2} & s_{1} b & =t_{1} b_{2} \\
a_{2} t_{2} & =a_{3} s_{3} & s_{2} b_{2}=t_{2} b_{3} \\
& \vdots & \vdots \\
a_{n} t_{n} & =a^{\prime} & s_{n} b_{n}=t_{n} b^{\prime}
\end{array}
$$

Received by the editors July 17, 1986.

1980 Mathematics Subject Classification (1985 Revision). Primary 20M10; Secondary 20M50.

Key words and phrases. Right regular band, left absolutely flat semigroup.

Research of the first author supported by Natural Sciences and Engineering Research Council grant A4494.

Research of second author supported by Natural Sciences and Engineering Research Council grant A9241. 
(where it is assumed that $S^{1}$ acts unitally on $A$ and $B$ ). The system of equalities above is called a scheme over $A$ and $B$ of length $n$ joining $(a, b)$ to $\left(a^{\prime}, b^{\prime}\right)$. From this description we see that a left $S$-set $B$ is flat if and only if, for every right $S$-set $A$, and every $a, a^{\prime} \in A, b, b^{\prime} \in B$ such that there exists a scheme over $A$ and $B$ joining $(a, b)$ to $\left(a^{\prime}, b^{\prime}\right)$, there exists a scheme (of possibly different length) over $a S^{1} \cup a^{\prime} S^{1}$ and $B$ joining $(a, b)$ to $\left(a^{\prime}, b^{\prime}\right)$. (See Lemma 2.2 of [1].)

In the sequel $S=\bigcup\left\{S_{\gamma} \mid \gamma \in \Gamma\right\}$ will denote a right regular band with its associated greatest semilattice decomposition ( $\Gamma$ is a semilattice, each $S_{\gamma}$ is a right zero semigroup, and $S_{\gamma} S_{\delta} \subseteq S_{\gamma \delta}$ for each $\gamma, \delta \in \Gamma$ ). If $F$ represents any subset of $S^{2}$ then $\theta_{R}(F)$ will indicate the smallest right congruence on $S$ containing $F$. The first condition (I) (for "insertion") in the following proposition is useful when considering schemes.

PROPOSITION. Let $S=\bigcup\left\{S_{\gamma} \mid \gamma \in \Gamma\right\}$ be a right regular band. The following statements are equivalent.

(I) For all $\alpha, \beta \in \Gamma, \alpha<\beta, u_{1}, v_{1}, \ldots, u_{m}, v_{m} \in S_{\beta}$ and for every $A \in$ Ens $-S$, $a_{1}, \ldots, a_{m+1} \in A$, if $a_{i} u_{i}=a_{i+1} v_{i}(1 \leq i \leq m)$ then $a_{i} w u_{i}=a_{i+1} w v_{i}(1 \leq i \leq m)$ for some $w \in S_{\alpha}$.

(C) For all $\alpha, \beta \in \Gamma, \alpha<\beta, u_{1}, v_{1}, \ldots, u_{m}, v_{m} \in S_{\beta}$, there exists $w \in S_{\alpha}$ such that $\left(w u_{i}, w v_{i}\right) \in \theta_{R}\left(\left(u_{1}, v_{1}\right), \ldots,\left(u_{m}, v_{m}\right)\right)(1 \leq i \leq m)$.

ProOF. (I) implies (C). Denote $\theta_{R}\left(\left(u_{1}, v_{1}\right), \ldots,\left(u_{m}, v_{m}\right)\right)$ by $\theta$, let $A=S / \theta$, suppose $\overline{u_{1}}$ represents the $\theta$-class containing $u_{1}$, note that $\overline{u_{1}} u_{i}=\overline{u_{1}} v_{i}(1 \leq i \leq m)$ in $A$ and apply (I). Then there exists $w \in S_{\alpha}$ such that $\overline{u_{1}} w u_{i}=\overline{u_{1}} w v_{i}(1 \leq i \leq m)$ and hence $\left(w u_{i}, w v_{i}\right) \in \theta(1 \leq i \leq m)$ since $u_{1} w=w$.

(C) implies (I). Suppose $A$ is a right $S$-set, $a_{1}, \ldots, a_{m+1}$ belong to $A$, and $a_{i} u_{i}=a_{i+1} v_{i}(1 \leq i \leq m)$. By right regularity it is clear that $a_{i} s=a_{j} s$ whenever $1 \leq i, j \leq m+1, s \in S_{\alpha} \cup S_{\beta}$, and that $a_{i} u_{i}=a_{i+1} u_{i}=a_{i} v_{i}=a_{i+1} v_{i}(1 \leq i \leq m)$. Let $F=\left\{\left(u_{1}, v_{1}\right),\left(v_{1}, u_{1}\right), \ldots,\left(u_{m}, v_{m}\right),\left(v_{m}, u_{m}\right)\right\}$. By $(C)$ there exists $w \in S_{\alpha}$ such that $\left(w u_{i}, w v_{i}\right) \in \theta_{R}(F)(1 \leq i \leq m)$. Now consider any $k \in\{1, \ldots, m\}$. We seek to prove $a_{k} w u_{k}=a_{k+1} w v_{k}$. If $w u_{k}=w v_{k}$ then $a_{k} w u_{k}=a_{k} u_{k} w u_{k}=$ $a_{k+1} v_{k} w u_{k}=a_{k+1} w u_{k}=a_{k+1} w v_{k}$. Otherwise there exist $y_{1}, \ldots, y_{n}, z_{1}, \ldots, z_{n} \in$ $S_{\beta}$ and $s_{1}, \ldots, s_{n} \in S^{1}$ such that $\left(y_{i}, z_{i}\right) \in F(1 \leq i \leq n)$ and

$$
\begin{aligned}
w u_{k} & =y_{1} s_{1} \\
z_{1} s_{1} & =y_{2} s_{2} \\
& \vdots \\
z_{n} s_{n} & =w v_{k} .
\end{aligned}
$$

For each $i \in\{1, \ldots, n\}$ there exists $j_{i} \in\{1, \ldots, m\}$ such that $\left(y_{i}, z_{i}\right) \in\left\{\left(u_{j_{i}}, v_{j_{i}}\right)\right.$, $\left.\left(v_{j_{i}}, u_{j_{i}}\right)\right\}$. Then $a_{k} w u_{k}=a_{k} y_{1} s_{1}=a_{j_{1}} y_{1} s_{1}=a_{j_{1}} z_{1} s_{1}=a_{j_{1}} y_{2} s_{2}=a_{j_{2}} y_{2} s_{2}=$ $a_{j_{2}} z_{2} s_{2}=\cdots=a_{j_{n}} z_{n} s_{n}=a_{j_{n}} w v_{k}=a_{k+1} w v_{k}$ as required.

Note that the second condition (C) (for "congruence") in the proposition above refers only to the semigroup $S$ itself and not to arbitrary $S$-sets. The following theorem, whose proof will appear in the next section, is the main result of this paper. (Recall that every left absolutely flat band is necessarily right regular.)

THEOREM. Every left absolutely flat band satisfies condition (C). 
Let $S=\bigcup\left\{S_{\gamma} \mid \gamma \in \Gamma\right\}$ be a right regular band and suppose that either $\Gamma$ is a chain or that $S$ is right normal. Then whenever $\alpha, \beta \in \Gamma, \alpha<\beta$, and $F \subseteq S_{\beta}^{2}$ it is true that $\theta_{R}(F) \cap S_{\alpha}^{2} \subseteq \Delta$. Hence we have

COROLLARY 1 [2]. If $S$ is a left absolutely flat normal band then the (nonidentity) structure maps of $S$ are constant.

Recall that every band is partially ordered by the relation $e \leq f$ if and only if $e f=e=f e$. We shall say that a right regular band $S=\bigcup\left\{S_{\gamma} \mid \alpha \in \Gamma\right\}$ has "the lower bound condition" if whenever $\alpha, \beta \in \Gamma$ and $\alpha<\beta$, every finite subset of $S_{\beta}$ has a lower bound in $S_{\alpha}$. This condition is equivalent to condition (C) in case $\Gamma$ is a chain.

COROLLARY 2. Let $S=\bigcup\left\{S_{\gamma} \mid \gamma \in \Gamma\right\}$ be a right regular band such that $\Gamma$ is a chain. Then $S$ is left absolutely flat if and only if $S$ has the lower bound condition.

PROOF. The necessity of the lower bound condition follows immediately from the theorem and the remark preceding Corollary 2.

Assume now that $S$ has the lower bound condition. Let

$$
\begin{aligned}
a & =a_{1} s_{1} & \\
a_{1} t_{1} & =a_{2} s_{2} & s_{1} b=t_{1} b_{2} \\
& \vdots & \vdots \\
a_{n} t_{n} & =a^{\prime} & s_{n} b_{n}=t_{n} b^{\prime}
\end{aligned}
$$

be a scheme of length $n$ joining $(a, b)$ to $\left(a^{\prime}, b^{\prime}\right)$ over $S$-sets $A$ and $B$ as described at the beginning of this section. We use induction on $n$ to show that a suitable replacement scheme over $a S^{1} \cup a^{\prime} S^{1}$ and $B$ can be found. When $n=1$ the scheme

$$
\begin{aligned}
a & =\left(a s_{1}\right) s_{1} & & \\
\left(a s_{1}\right) t_{1} & =\left(a^{\prime} s_{1}\right) t_{1} & s_{1} b & =t_{1} b^{\prime} \\
\left(a^{\prime} s_{1}\right) s_{1} & =a^{\prime} s_{1} & t_{1} b^{\prime} & =s_{1} b \\
a^{\prime} t_{1} & =a^{\prime} & s_{1} b & =t_{1} b^{\prime}
\end{aligned}
$$

is appropriate (in fact these equalities hold for any right regular band). Assume now that $n>1$ and that all schemes of length less than $n$ can be replaced. Suppose $s_{1} \in S_{\alpha}, t_{1} s_{2} \cdots t_{n-1} s_{n} \in S_{\beta}$, and $t_{n} \in S_{\gamma}$. If $\alpha \geq \beta$ or $\gamma \geq \beta$ then $a_{i} t_{i} \in a S \cup a^{\prime} S$ for some $i \in\{1, \ldots, n-1\}$ and the scheme may be resolved into two shorter schemes. The proof can then be completed as shown in [2, proof of Theorem 2.4]. We therefore assume $\alpha, \gamma<\beta$ and without loss of generality suppose that $\alpha \geq \gamma$. The lower bound condition can be used to find an element $l \in S_{\alpha}$ such that $l t_{1}=l s_{2}=\cdots=l t_{n-1}=l s_{n}=l$. Then an appropriate replacement scheme is

$$
\begin{aligned}
a & =a s_{1} & & \\
a l & =a l & & s_{1} b=l b_{n} \\
a t_{n} & =a^{\prime} & & l b_{n}=t_{n} b^{\prime}
\end{aligned}
$$

and the proof is complete.

In general the lower bound condition of this corollary is not necessary for a right regular band to be left absolutely flat, as the following example shows. Consider 
the band $S=S_{\alpha} \cup S_{\beta} \cup S_{\gamma}$ where $S_{\alpha}=\{a, b\}, S_{\beta}=\{c\}$, and $S_{\gamma}=S_{\alpha \beta}=\{d, e\}$ with table

\begin{tabular}{l|lllll} 
& $a$ & $b$ & $c$ & $d$ & $e$ \\
\hline$a$ & $a$ & $b$ & $d$ & $d$ & $e$ \\
$b$ & $a$ & $b$ & $e$ & $d$ & $e$ \\
$c$ & $d$ & $e$ & $c$ & $d$ & $e$ \\
$d$ & $d$ & $e$ & $d$ & $d$ & $e$ \\
$e$ & $d$ & $e$ & $e$ & $d$ & $e$
\end{tabular}

Note that $\{a, b\}$ does not have a lower bound in $S_{\gamma}$ and that $S$ has condition (C). The fact that this semigroup is left absolutely flat is a consequence of the following more general result. If $S=\bigcup\left\{S_{\gamma} \mid \gamma \in \Gamma\right\}$ is a right regular band satisfying (C) and if every chain in $\Gamma$ has no more than two elements then $S$ is left absolutely flat. For simplicity our proof of this fact assumes $\Gamma$ has exactly two maximal elements $\alpha$ and $\beta$ (as is the case in the example above). Suppose

$$
\begin{array}{rlrl}
a & =a_{1} s_{1} & \\
a_{1} t_{1} & =a_{2} s_{2} & s_{1} b & =t_{1} b_{2} \\
& \vdots & \vdots \\
a_{n} t_{n} & =a^{\prime} & s_{n} b_{n}=t_{n} b^{\prime}
\end{array}
$$

is a scheme of length $n$ over $S$-sets $A$ and $B$ joining $(a, b)$ to $\left(a^{\prime}, b^{\prime}\right)$. If $n=1$ the replacement scheme of length 3 displayed in the proof of Corollary 2 suffices. Now assume $n>1$. Without loss of generality we may assume $t_{1}, s_{2}, \ldots, t_{n-1}, s_{n}$ belong to $S_{\alpha} \cup S_{\beta}$ and in fact, $t_{i}, s_{i+1} \in S_{\alpha}$ or $t_{i}, s_{i+1} \in S_{\beta}$ for each $i \in\{1, \ldots, n-1\}$, because otherwise $a_{i} t_{i} \in a S \cup a^{\prime} S$ for some $i$ and induction may be used (as in the proof of Corollary 2). Suppose $t_{1} \in S_{\alpha}$. Then $s_{1} \in S_{\beta} \cup S_{\gamma}$ (since, if not, $\left.a_{1} t_{1} \in a S\right)$. Let $i$ be the largest index such that $t_{j} \in S_{\alpha}$ for all $j, 1 \leq j \leq i$. If $i=n, a_{n} s_{n} \in a^{\prime} S$ would result and therefore we assume $i<n$. Because we have condition (C) and, hence, (I), there exists $w \in S_{\gamma}$ such that

$$
\begin{array}{rlrl}
a & =a_{1} s_{1} & \\
a_{1}\left(w t_{1}\right) & =a_{2}\left(w s_{2}\right) & s_{1} b & =\left(w t_{1}\right) b_{2} \\
& \vdots & \vdots \\
a_{i}\left(w t_{i}\right) & =a_{i}\left(w s_{i+1}\right) & \left(w s_{i}\right) b_{i} & =\left(w t_{i}\right) b_{i+1} \\
a_{i} t_{i+1} & =a_{i+1} s_{i+1} & \left(w s_{i+1}\right) b_{i+1} & =t_{i+1} b_{i+2} \\
& \vdots & \vdots \\
a_{n} t_{n} & =a^{\prime} & s_{n} b_{n} & =t_{n} b^{\prime} .
\end{array}
$$

Note that $s_{1} b=t_{1} s_{1} b=w t_{1} s_{1} b=w t_{1} b_{2}$ and the equality $\left(w s_{i+1}\right) b_{i+1}=t_{i+1} b_{i+2}$ follows similarly. We now have a scheme of length $n$ over the $S$-sets $A$ and $B$ joining $(a, b)$ to $\left(a^{\prime}, b^{\prime}\right)$ in which $a_{1}\left(w t_{1}\right) \in a S$. Induction may be used to complete the proof. (If $t_{1} \in S_{\beta}$ a similar proof serves.)

3. Proof of the theorem. Let $S=\bigcup\left\{S_{\gamma} \mid \alpha \in \Gamma\right\}$ be a left absolutely flat band. Then $S$ is right regular (i.e. each $S_{\gamma}$ is a right zero semigroup). Suppose $\alpha, \beta \in \Gamma, \alpha<\beta$, and suppose $F=\left\{\left(u_{1}, v_{1}\right), \ldots,\left(u_{m}, v_{m}\right)\right\}$ is a finite subset of $S_{\beta}^{2}$. 
We will first show that condition (C) holds under the assumption $\theta_{R}(F) \cap S_{\alpha}^{2} \subseteq \Delta$; i.e. we will prove that, in this situation, there exists $w \in S_{\alpha}$ such that $w u_{i}=$ $w v_{i}(1 \leq i \leq m)$. Since all filters in $S$ are left absolutely flat (see [1]) we may suppose without loss of generality that $\alpha$ is the smallest element of $\Gamma$. Because $\theta_{R}(F) \cap S_{\alpha}^{2} \subseteq \Delta$ there exists an embedding $S_{\alpha} \hookrightarrow S / \theta_{R}(F)$ of right $S$-sets. Select any $l \in S_{\alpha}$. Let $Y=S^{1} y \dot{\cup} S^{1} y_{1} \dot{\cup} \cdots \dot{\cup} S^{1} y_{m} \dot{\cup} S^{1} y^{\prime}$ be the free left $S$-set on free generators $\left\{y, y_{1}, \ldots, y_{m}, y^{\prime}\right\}$, denote by $\psi$ the congruence on $Y$ generated by the set

$$
G=\left\{\left(l y, u_{1} y_{1}\right),\left(v_{1} y_{1}, u_{2} y_{2}\right), \ldots,\left(v_{m-1} y_{m-1}, u_{m} y_{m}\right),\left(v_{m} y_{m}, l y^{\prime}\right)\right\}
$$

and let $B$ represent the left $S$-set $Y / \psi$. Note that $\bar{l} \otimes \bar{y}=\bar{l} \otimes \overline{y^{\prime}}$ in $S / \theta_{R}(F) \otimes B$ :

$$
\begin{aligned}
\bar{l} \otimes \bar{y} & =\overline{u_{1} l} \otimes \bar{y}=\overline{u_{1}} \otimes \overline{l y}=\overline{u_{1}} \otimes \overline{u_{1} y_{1}}=\overline{u_{1}} \otimes \overline{y_{1}} \\
& =\overline{v_{1}} \otimes \overline{y_{1}}=\overline{v_{1}} \otimes \overline{v_{1} \bar{y}_{1}}=\overline{v_{1}} \otimes \overline{u_{2} y_{2}}=\overline{v_{1} u_{2}} \otimes \overline{y_{2}}=\overline{u_{2}} \otimes \overline{y_{2}} \\
& =\cdots \overline{u_{m}} \otimes \overline{y_{m}}=\overline{v_{m}} \otimes \overline{y_{m}}=\overline{v_{m}} \otimes \overline{v_{m} y_{m}}=\overline{v_{m}} \otimes \overline{l y^{\prime}}=\overline{v_{m} l} \otimes \overline{y^{\prime}} \\
& =\bar{l} \otimes \overline{y^{\prime}} .
\end{aligned}
$$

Since $B$ is flat it follows that $l \otimes \bar{y}=l \otimes \overline{y^{\prime}}$ in $S_{\alpha} \otimes B$, hence also in $S^{1} \otimes B \cong B$. Thus, $\overline{l y}=\overline{l y^{\prime}}$ in $B$, or $\left(l y, l y^{\prime}\right) \in \psi$. This means there exist $s_{1}, s_{2}, \ldots, s_{n} \in S^{1}$, $x_{1}, x_{2}, \ldots, x_{n} \in Y$, and $z_{1}, z_{2}, \ldots, z_{n} \in Y$ such that

$$
\begin{aligned}
l y & =s_{1} x_{1} \\
s_{1} z_{1} & =s_{2} x_{2}
\end{aligned}
$$

$$
\begin{aligned}
s_{n-1} z_{n-1} & =s_{n} x_{n} \\
s_{n} z_{n} & =l y^{\prime},
\end{aligned}
$$

where $\left(x_{i}, z_{i}\right) \in G \cup G^{-1}(1 \leq i \leq n)$.

Assume $n$ is the smallest positive integer for which such a scheme exists. We now prove that, in fact, $\left(x_{i}, z_{i}\right) \in G(1 \leq i \leq n)$. Since $l y=s_{1} x_{1}, x_{1}=l y$ and hence, $\left(x_{1}, z_{1}\right) \in G$. Suppose (by way of contradiction) there exists an index $j$ such that $\left(x_{j}, z_{j}\right) \in G^{-1}$. Let $i$ be the smallest such index. Then since $s_{i-1} z_{i-1}=s_{i} x_{i}$, $z_{i-1}=x_{i}$ because $\left(x_{i-1}, z_{i-1}\right) \in G$. If $z_{i-1}=u_{1} y_{1}$ then $s_{i} z_{i}=s_{i} l y=l y$ and, consequently, $l y$ and $l y^{\prime}$ may be joined by a shorter scheme (contradiction). If there exists $j(2 \leq j \leq m)$ such that $z_{i-1}=u_{j} y_{j}$ then $s_{i-1} z_{i-1}=s_{i} x_{i}$ becomes $s_{i-1} u_{j} y_{j}=s_{i} u_{j} y_{j}$ which shows $s_{i-1} u_{j}=s_{i} u_{j}$ and, hence, $s_{i-1} v_{j-1}=s_{i} v_{j-1}$. It now follows that $s_{i-1} x_{i-1}$ equals $s_{i} z_{i}$ and therefore, deletion of the equation $s_{i-1} z_{i-1}=s_{i} x_{i}$ yields a shorter scheme joining $l y$ and $l y^{\prime}$ (contradiction). If $z_{i-1}=$ $l y^{\prime}$ then $s_{i} x_{i}=s_{i} l y^{\prime}=l y^{\prime}$ and again, $l y$ and $l y^{\prime}$ are connected by a shorter scheme (contradiction). Therefore $(\Sigma)$ is in fact the scheme

$$
\begin{aligned}
l y & =s_{1} l y \\
s_{1} u_{1} y_{1} & =s_{2} v_{1} y_{1} \\
s_{2} u_{2} y_{2} & =s_{3} v_{2} y_{2} \\
& \vdots \\
s_{m} u_{m} y_{m} & =s_{m+1} v_{m} y_{m} \\
s_{m+1} l y^{\prime} & =l y^{\prime} .
\end{aligned}
$$


Consequently, in $S$

$$
\begin{aligned}
l & =s_{1} l \\
s_{1} u_{1} & =s_{2} v_{1} \\
s_{2} u_{2} & =s_{3} v_{2} \\
& \vdots \\
s_{m} u_{m} & =s_{m+1} v_{m} \\
s_{m+1} l & =l .
\end{aligned}
$$

Let $w=l s_{1}$. Then for each $i(1 \leq i \leq m), w u_{i}=l s_{1} u_{i}=l s_{1} u_{1} u_{i}=l s_{2} v_{1} u_{i}=$ $l s_{2} u_{2} u_{i}=\cdots=l s_{i} u_{i} u_{i}=l s_{i} u_{i}=l s_{i+1} v_{i}$ and hence, $w u_{i}=w v_{i}$ as required.

We return now to the general situation (in which the relation $\theta_{R}(F) \cap S_{\alpha}^{2} \subseteq \Delta$ is not assumed). Consider the filter $S_{[\alpha)}=\bigcup\left\{S_{\gamma} \mid \gamma \geq \alpha\right\}$, which is also left absolutely flat. The equivalence relation $\theta=\left(\theta_{R}(F) \cap S_{\alpha}^{2}\right) \cup \Delta$ is easily seen to be a (two-sided) congruence on $S_{[\alpha)}$. The previous argument may be applied to the left absolutely flat right regular band $S_{[\alpha)} / \theta$ to eventually obtain $w$ in $S_{\alpha}$ such that $\left(w u_{i}, w v_{i}\right) \in \theta_{R}(F)(1 \leq i \leq m)$.

4. Concluding remarks. If $S$ is a right regular band satisfying condition (C) then it is possible to prove that every scheme of length 2

$$
\begin{array}{rlrl}
a & =a_{1} s_{1} & \\
a_{1} t_{1} & =a_{2} s_{2} & & s_{1} b=t_{1} b_{2} \\
a_{2} t_{2} & =a^{\prime} & s_{2} b_{2}=t_{2} b^{\prime}
\end{array}
$$

joining $(a, b)$ to $\left(a^{\prime}, b^{\prime}\right)$ over $S$-sets $A$ and $B$ (see $\left.\S 2\right)$ may be replaced by a (symmetric) scheme of length 14 joining $(a, b)$ to $\left(a^{\prime}, b^{\prime}\right)$ over $a S^{1} \cup a^{\prime} S^{1}$ and $B$. In spite of this and the fact that condition $(C)$ implies left absolute flatness in special cases, the authors doubt that this implication holds for right regular bands in general. The investigation will be continued in a subsequent paper.

\section{REFERENCES}

1. S. D. Bulman-Fleming and K. McDowell, Absolutely flat semigroups, Pacific J. Math. 107 (1983), 319-333.

2. $\_$, Left absolutely flat generalized inverse semigroups, Proc. Amer. Math. Soc. 94 (1985), 553-561.

3. M. Kil'p, Left completely flat monoids that are unions of groups, Tartu Riikl. Ül. Toimetised No. 556, Trudy Mat. Meh. (1981), 33-37. (Russian; Estonian and English summaries).

DEPARTMENT OF MATHEMATICS, WilfRid LAURIER UNIVERSity, Waterloo, ONTARIO, CANADA N2L 3C5 